\title{
Avaliação eletromanométrica do esfíncter superior do esôfago em portadores da forma indeterminada da doença de Chagas
}

\author{
Manometric evaluation of upper esophageal sphincter in patients with \\ indeterminate form of Chagas' disease
}

\author{
Eduardo Crema1, Renata Mônica de Oliveira², Ana Márcia Werneck², \\ Ricardo Pastore ${ }^{1}$, Aiodair Martins Junior ${ }^{1}$ e Alex Augusto Silva ${ }^{1}$
}

\begin{abstract}
RESUMO
Objetivou-se avaliar as alterações do esfíncter superior esofágico pela eletromanometria em 37 pacientes portadores da forma clínica indeterminada da doença de Chagas. Foram encontrados 18 (48,6\%) pacientes portadores de ondas sincrônicas. A média das pressões máximas do esfíncter foi significativamente maior entre os portadores de ondas sincrônicas. Assim, alguns indivíduos portadores da forma indeterminada da doença de Chagas possuem alterações funcionais caracterizadas pelo aumento da pressão do esfíncter superior do esôfago, que podem ser detectadas à eletromanometria.
\end{abstract}

Palavras-chaves: Doença de Chagas. Forma indeterminada. Eletromanometria. Esfíncter superior do esôfago.

\begin{abstract}
The objective was to study the disorders of upper esophageal sphincter in 37 patients with indeterminate clinical form of Chagas' disease. Eighty (48.6\%) patients with synchronic waves were found. The average maximum pressure value of the upper esophageal sphincter was significantly higher in the synchronic group. Subjects with indeterminate clinical form of Chagas' disease may have functional disorders demonstrated by an increase in the pressure of the upper esophageal sphincter.
\end{abstract}

Key-words: Chagas' disease. Indeterminated form. Manometric study. Upper esophageal sphincter.

A doença de Chagas cursa com degeneração e conseqüente redução do número de neurônios do plexo mioentérico esofágico, ocasionando alterações funcionais e morfológicas características da doença ${ }^{7}$.

Os indivíduos que apresentam a forma indeterminada da doença de Chagas se caracterizam por apresentarem reação imunológica positiva para a enfermidade, ao mesmo tempo em que não se constatam sintomas clínicos ou alterações eletrocardiográficas ou radiológicas nos exames cardíaco e contrastados do esôfago e cólon ${ }^{11}{ }^{12}$.

0 conhecimento atual nos permite afirmar, que testes mais sensíveis podem detectar alterações precoces em pacientes com a forma indeterminada da doença de Chagas. Em relação à esofagopatia, a eletromanometria torna-se um importante exame permitindo a detecção de alterações funcionais precocemente $^{39}$, além de orientar quanto a melhor opção para o tratamento cirúrgico ${ }^{2}$.

O esfíncter superior do esôfago (ESE) é considerado um esfíncter funcional, formado principalmente pelo músculo cricofaríngeo, controlado pelo sistema nervoso central através do nervo vago em resposta a estímulos originados na orofaringe e no esôfago ${ }^{8}$.

Sabe-se que a desnervação decorrente da doença de Chagas é a responsável pela redução da amplitude de contração e pela alteração da peristalse harmônica do corpo esofágico ${ }^{1391314}$ que conduz o bolo alimentar ao estômago. Estas alterações, são dependentes da forma como a doença se apresenta. Muitos estudos têm procurado demonstrar alterações funcionais da esofagopatia chagásica através da análise eletromanométrica do esôfago ${ }^{14610}$. Entretanto, pouco

\footnotetext{
1. Disciplina de Cirurgia do Aparelho Digestivo da Universidade Federal do Triângulo Mineiro, Uberaba, MG. 2. Acadêmica do Curso de Medicina da Universidade Federal do Triângulo Mineiro, Uberaba, MG.

Apoio: FAPEMIG

Endereço para correspondência: Dr. Eduardo Crema, R. Marcos Lombardi 305, 38050-170 Uberaba-MG

Fax: $55343315-4500$

e-mail: eduardocremafmtm@mednet.com.br

Recebido para publicação em 2/12/2005

Aceito em 28/5/2004
} 
se estudou sobre as alterações específicas que ocorrem no ESE na doença de Chagas ${ }^{5}$. Este estudo objetivou analisar as alterações eletromanométricas do esfíncter superior do esôfago em portadores da forma indeterminada da doença de Chagas.

\section{PACIENTES E MÉTODOS}

Foram incluidos neste estudo 37 pacientes portadores da doença de Chagas, comprovados por pelo menos dois testes sorológicos positivos (imunofluorescência indireta, ELISA ou hemoaglutinação) e que tinham eletrocardiograma e exames cardiológicos do coração, esôfago e colon normais.

0s 37 pacientes foram submetidos à eletromanometria esofágica através de uma sonda plástica introduzida pelo orifício nasal até o estômago. A sonda é composta por 6 canais (3 orifícios na extremidade mais distal, e os outros 3 com espaçamento de $5 \mathrm{~cm}$ entre eles) por onde perfundiu-se água destilada a uma velocidade constante de $0,6 \mathrm{ml} / \mathrm{min} /$ canal. Após a comprovação, de que todos os canais se encontravam situados no abdome, a sonda foi retirada de forma intermitente, centímetro a centímetro. Ao final do procedimento, foi possível analisar simultaneamente a localização, extensão e pressão máxima do esfíncter esofágico superior. Qualquer obstáculo ao fluxo de água era traduzido como variação da pressão intraluminar. 0 registro das alterações pressóricas foi feito através de um polígrafo e decodificado por um software próprio (Polygram for Windows versão 2.02), possibilitando a análise da pressão do esfíncter superior do esôfago. A pressão da ESE foi medida por três vezes em cada paciente, e a média aritmética das três medidas foi empregada como valor de referência de cada paciente. Os dados obtidos foram analisados por um programa estatístico, aplicando-se o teste t-student.

Este ensaio foi submetido e aprovado pelo Comitê de ética em pesquisa da Faculdade de Medicina do Triângulo Mineiro, e os pacientes nele envolvidos mantêm-se sob acompanhamento médico-ambulatorial e assistência social frequientes.

\section{RESULTADOS}

Após analise manométrica do esôfago, os pacientes foram divididos em 2 grupos: grupo 1, constituído por 19 (51,4\%) pacientes que apresentavam ondas assincrônicas e grupo 2, constituído de 18 (48,6\%) pacientes em que foram detectadas ondas sincrônicas. Não se observou diferenças estatisticamente significantes entre os grupos quanto idade e sexo, sendo, portanto os grupos considerados homogêneos.

A comparação das médias das pressões máximas do esfíncter superior do esôfago nos pacientes portadores de ondas sincrônicas ao teste manométrico foi significativamente maior $(204,17 \mathrm{mmHg})$ que a média encontrada nos portadores de ondas assincrônicas $(142,44 \mathrm{mmHg})$ (Figura 1).

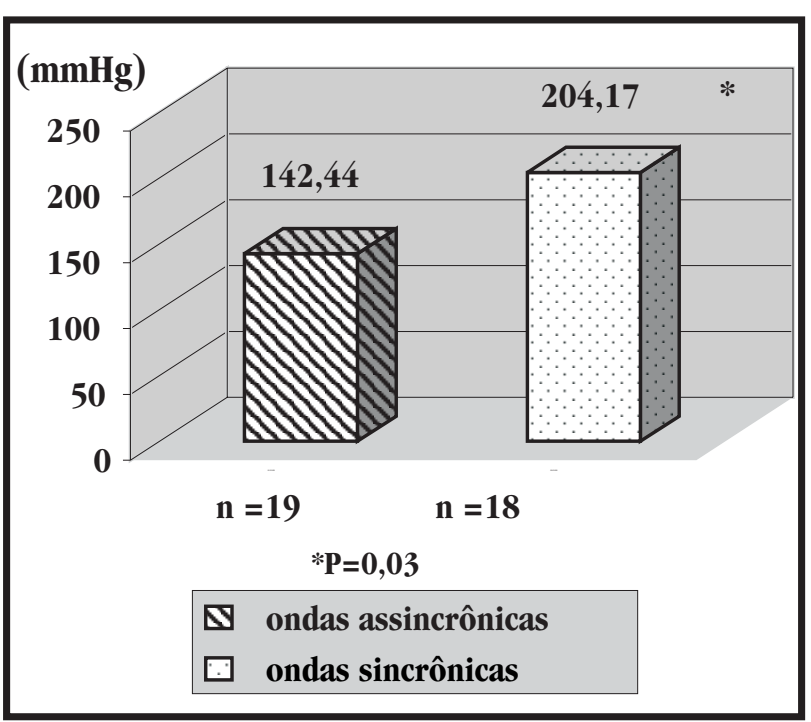

Figura 1 - Comparação das médias das pressões máximas do esfíncter superior entre pacientes portadores da forma indeterminada da doença de Chagas com ondas sincrônicas e assincrônicas ao teste manométrico.

\section{DISCUSSÃO}

$\mathrm{Na}$ investigação das alterações funcionais decorrentes da desnervação esofágica que ocorre na doença de Chagas, alguns estudos já demonstraram uma diminuição total ou parcial do relaxamento do esfíncter inferior do esôfago, acompanhada do sincronismo das ondas de contração do corpo esofágico, chamada por alguns autores de aperistalse ${ }^{4513}$. Poucos estudos, porém, avaliaram a alteração funcional do esôfago em pacientes com a forma indeterminada da doença de Chagas.

Dantas $^{4}$ em 2000 comparou as medidas de pressão do ESE em indivíduos voluntários sadios, chagásicos e portadores de acalasia idiopática sem, porém avaliar o grupo da forma indeterminada. Sua conclusão foi a de que os indivíduos chagásicos e que apresentaram aspecto radiológico normal do esôfago demostraram pressões estatisticamente mais elevadas do ESE.

Neste estudo, optou-se por não utilizar a comparação com grupo controle sadio, como o fez Dantas ${ }^{4}$, uma vez que os padrões de normalidade da fisiologia esofágica à eletromanometria já estão amplamente estabelecidos ${ }^{16}$.

Nesse ensaio observou-se que dentro do grupo de pacientes com a forma indeterminada da doença de Chagas, existem dois tipos de indivíduos. Aquele que apresenta alteração da eletrofisiologia esofágica, traduzida pela presença de ondas peristálticas sincrônicas do corpo esofágico, e aqueles que apresentam a fisiologia normal do esôfago (ondas assincrônicas).

A existência de 18 (48,6\%) indivíduos portadores da forma indeterminada com esofagopatia inicial condiz com a conceituação de Ribeiro e Rocha ${ }^{15}$, Macêdo ${ }^{12}$ e Marin-Neto e cols $^{14}$ de que tais indivíduos quando submetidos a testes mais sensíveis podem demonstrar alterações fisiológicas não detectáveis nos testes usuais.

O grupo de indivíduos com ondas sincrônicas evidenciou maiores pressões do esfíncter esofágico superior, com 
significância estatística, quando comparado ao grupo cujo corpo esofágico apresentou ondas assincrônicas, isto talvez se deva a resposta compensatória do músculo cricofaríngeo (músculo estriado) a diminuição do peristaltismo esofágico.

Dantas 5 apesar de não ter estudado especificamente o grupo da forma indeterminada, obteve resultados semelhantes quando comparou a pressão do ESE em pacientes chagásicos com e sem dilatação radiográfica, voluntários normais e portadores de acalásia idiopática. Em seu estudo, notou que pacientes chagásicos e sem alteração radiológica apresentavam maior pressão do esfíncter superior do esôfago, quando comparados aos demais.

A eletromanometria é um método sensível na avaliação das alterações eletrofisiológicas primitivas do esôfago dos indivíduos com reação imunológica positiva para doença de Chagas e sem alterações anátomo-clínicas. Neste grupo evidenciou-se aumento da pressão do ESE, talvez como primeiras alterações fisiológicas em relação ao esôfago normal.

\section{REFERÊNCIAS BIBLIOGRÁFICAS}

1. Crema E, Cruvinel LAF, Werneck AM, Oliveira RM, Silva AA. Correlação manométrico-radiológica no tratamento cirúrgico do megaesôfago chagásico. Revista da Sociedade Brasileira de Medicina Tropical 36: 665-669, 2003.

2. Crema E, Oliveira RM, Werneck AM, Cruvinel LAF, Silva AA - Estudo eletromanométrico do esôfago em portadores da doença de Chagas em sua forma indeterminada. Revista do Colégio Brasileiro de Cirurgiões 31: 107-111, 2004.

3. Dantas RO. Vigorous achalasia in Chagas disease. Journal of Clinical Gastroenterology 28:245-248, 1999.

4. Dantas R0. Upper esophageal sphincter pressure in patients with Chagas' disease and primary achalasia. Brazilian Journal of Medical and Biological Research 33: 545-550, 2000.
5. Dantas R0, Deghaide NH, Donadi EA. Esophageal manometric and radiologic findings in asympthomatic subjects with Chagas' disease. Rozhledy Chirurgii 81:183-187, 2002.

6. Dantas RO, Godoy RA, Padovan W. A contratilidade do esôfago na esofagopatia chagásica. Gastrenterologia Endoscopia Digestiva 2: 26-28, 1983.

7. Ferreira MS, Lopes ER, Chapadeiro E. Doença de Chagas. In: Veronesi R, Focaccia R (eds) Tratado de Infectologia, $4^{\mathrm{a}}$ edição, São Paulo, Atheneu, vol 2, p.1175-1211, 1999.

8. Kahrilas PJ, Dodds WJ, Dent J, Logemann JÁ, Shaker R. Upper esophageal sphincter function during deglutition. Gastroenterology 95:52,1988.

9. Leme EM, Domingues GR, Pereira VL, Firman CG, Pantoja J. Lower esophageal sphincter pressure in idiophathic achalasia and Chagas disease related achalasia. Acta Gastroenterológica Latinoamericana 24: 105-111 , 1994.

10. Lovecek M, Duda M, Gryga A, Dlouhy M, Herman J, Svach I. Manometry in esophageal achalasia. Diseases of Esophagus 14: 232-234, 2001.

11. Oliveira RB , Rezende JF, Dantas RO, Iazigi N. The Spectrum of Esophageal Motor Disorders in Chagas' Disease. American Journal of Gastroenterology 90: 1119-1124, 1995

12. Pinotti HW, Felix VN, Zilberstein B, Cecconello I. Surgery complications of Chagas disease: megaesophagus, achalasia of the pylorus and cholelithiasis. World Journal of Surgery 15:198-204,1991.

13. Macêdo V. Indeterminate form of Chagas disease. Memórias do Instituto Oswaldo Cruz 94 (supl 1): 311-316,1999.

14. Marin-Neto JA, Almeida-Filho OC, Pazin-Filho A, Maciel BC. Forma indeterminada da Moléstia de Chagas. Proposta de novos critérios de caracterização e perspectivas de tratamento precoce da cardiomiopatia. Arquivos Brasileiros de Cardiologia 79: 623-627, 2002.

15. Ribeiro AL, Rocha MO. Indeterminate form of Chagas disease: considerations about diagnosis and prognosis. Revista da Sociedade Brasileira de Medicina Tropical 31: 301-314, 1998.

16. Richter JE, Wu WC, Johns DN, Blackwell JN, Nelson JL, Castell JA, Castell DO. Esophageal manometry in 95 healthy adult volunteers: variability of pressures with age and frequency of "abnormal"contractions. Digestive Diseases and Sciences 32: 583-592, 1987. 